\title{
tomo3d: a new 3-D joint refraction and reflection travel-time tomography code for active-source seismic data
}

\author{
A. Meléndez (1), J. Korenaga (2), V. Sallarès (3), and C. R. Ranero (4) \\ (1) Barcelona Center for Subsurface Imaging, ICM, CSIC, Barcelona, Spain (melendez@ cmima.csic.es), (2) Department of \\ Geology and Geophysics, Yale University, CT, USA, (3) Barcelona Center for Subsurface Imaging, UTM, CSIC, Barcelona, \\ Spain, (4) Barcelona Center for Subsurface Imaging, ICREA at ICM, CSIC, Barcelona, Spain
}

We present the development state of tomo3d, a code for three-dimensional refraction and reflection travel-time tomography of wide-angle seismic data based on the previous two-dimensional version of the code, tomo $2 \mathrm{~d}$. The core of both forward and inverse problems is inherited from the 2-D version. The ray tracing is performed by a hybrid method combining the graph and bending methods. The graph method finds an ordered array of discrete model nodes, which satisfies Fermat's principle, that is, whose corresponding travel time is a global minimum within the space of discrete nodal connections. The bending method is then applied to produce a more accurate ray path by using the nodes as support points for an interpolation with beta-splines. Travel time tomography is formulated as an iterative linearized inversion, and each step is solved using an LSQR algorithm. In order to avoid the singularity of the sensitivity kernel and to reduce the instability of inversion, regularization parameters are introduced in the inversion in the form of smoothing and damping constraints.

Velocity models are built as 3-D meshes, and velocity values at intermediate locations are obtained by trilinear interpolation within the corresponding pseudo-cubic cell. Meshes are sheared to account for topographic relief. A floating reflector is represented by a 2-D grid, and depths at intermediate locations are calculated by bilinear interpolation within the corresponding square cell. The trade-off between the resolution of the final model and the associated computational cost is controlled by the relation between the selected forward star for the graph method (i.e. the number of nodes that each node considers as its neighbors) and the refinement of the velocity mesh.

Including reflected phases is advantageous because it provides a better coverage and allows us to define the geometry of those geological interfaces with velocity contrasts sharp enough to be observed on record sections. The code also offers the possibility of including water-layer multiples in the modeling, whenever this phase can be followed to greater offsets than the primary phases. This increases the quantity of useful information in the data and yields more extensive and better constrained velocity and geometry models.

We will present results from benchmark tests for forward and inverse problems, as well as synthetic tests comparing an inversion with refractions only and another one with both refractions and reflections. 